Z. Klin. Chem. Klin. Biochen.

10. Jg. 1972 , S. $430-433$

\title{
Quantitative enzymatische Bestimmung der Galaktokinaseaktivität im Erythrocytenhämolysat ${ }^{1}$ )
}

\author{
Von M. Gesche und F. Menne \\ Aus der Abteilung firr Stoffivecbselforscbung (Vorstand: Professor Dr. F. Menne) \\ des Physiologisch-Chemischen Instituts der Universität Münster
}

(Eingegangen am 14. März/9. Juni 1972)

Die Bestimmung der Galaktokinaseaktivität (EC 2.7.1.6) ist für die Diagnose der genetisch bedingten Stoffwechselstörung, des Galaktokinasemangels, unentbehrlich. Der bisher sicherste Weg der quantitativen Bestimmung der Galaktokinaseaktivität von NG, DoNNELL und BERGREN arbeitet mit dem Isotop ${ }^{14} \mathrm{C}$ und ist somit aufwendig und zeitraubend. Diese Schwierigkeiten vermeidet die vorliegende Methode, die sich - wie eingehende Kontrolluntersuchungen zeigten - durch große Genauigkeit und bequeme Handhabung auszeichnet. Die vorliegende quantitative Bestimmungsmethode der Galaktokinaseaktivität läuft folgendermaßen ab: Unter definierten Bedingungen wird Galaktose durch Galaktokinase phosphoryliert. Nach Abstoppen der Reaktion wird die nichtphosphorylierte Galaktose mit Hilfe der Galaktosedehydrogenase (EC 1.1.1.48) mit NAD ${ }^{+}$) bestimmt. Die gebildete NADH-Menge entspricht der umgesetzten Galaktosekonzentration.

\section{The quantitative determination of galactokinase activity in erytbrocyte baemolysates}

The determination of galactokinase activity (EC 2.7.1.6) is essential for the diagnosis of the genetically determined metabolic disorder of galactokinase deficiency. Hitherto, the most reliable method has been that of NG, DONNELL and BERGREN, which employs ${ }^{14} \mathrm{C}$ and is consequently expensive and time consuming. The present method does not have taesc disadvantages and extensive control studies show that it is very accurate and easy to operate. Under standardized conditions, galactose is phosphorylated by galactokinase. The reaction is stopped and non-phosphorylated galactose is determined with the aid of galactose dehydrogenase (EC1.1.1.48) and NAD+). The measured parameter is the quantity of $\mathrm{NADH}$, which corresponds to the concentration of free galactose available for dehydrogenation, i. e. not phosphorylated by galactokinase.

50 Jahre nach der Erstbeschreibung der Galaktosämie wurde die ursächlich enzymatische Störung - Uridylyltransferasemangel (EC 2.7.7.12) - 1956 von LELorR, KALCKAR et al. (1) gefunden.

1965 beschrieb Gitzelmann $(2,3)$ zum ersten Mal den Galaktokinasemangel.

Der Nachweis des Kinasemangels wurde bisher mit dem Isotopen ${ }^{14} \mathrm{C}$-Verfahren nach NG, DoNNELl und BERGREN (4) erbracht. Die Inkubation dauert bei $37^{\circ} \mathrm{C}$ in einem konst. Wasserbad 30 Minuten.

Der Bestimmungsansatz besteht aus:

$0,56 \mu \mathrm{mol}\left[1-{ }^{14} \mathrm{C}\right]$ Galaktose (mit der spezifischen Aktivität von $3,32 \mu \mathrm{Ci} / \mathrm{mg}$ nach Angaben des National Bureau of Standards); $4 \mu \mathrm{mol}$ ATP; $0,08 \mu \mathrm{mol} \mathrm{MgSO}_{4} ; 150 \mu \mathrm{mol}$ Trispuffer beim pH 7,0 und $0,4 \mathrm{ml} 50$ proz. Hämolysat in einem Totalvolumen von $1 \mathrm{ml}$. Die Teste werden doppelt gefahren. Es folgen Chromatographie und Scintillationszählung.

Die Methode ist sehr aufwendig, zeitraubend und erfordert eine große Erfahrung.

Zur Vermeidung dieser Schwierigkeiten wurde die vorliegende enzymatische Methode entwickelt.

1) Durchgeführt mit freundlicher Unterstützung des Ministerpräsidenten des Landes Nordrhein-Westfalen - Landesamt für Forschung.

\section{Methodik}

\section{Prinzip}

Das Prinzip dieser enzymatischen Methode beruht auf dem Verbrauch von Galaktose. Unter den Ansatzbedingungen wird Galaktose durch die Galaktokinase (EC 2.7.1.6) phosphoryliert. Nach Abstoppen der Reaktion wird die nichtphosphorylierte Galaktose mit Hilfe det Galaktose-Dehydrogenase (EC 1.1.1.48) im optischen Test bestimmt. Die gebildete NADH-Menge ist der umgesetzten Galaktose-Menge äquivalent.

Die Reaktion läuft folgendermaßen ab:

1. $\mathrm{Gal}+\mathrm{ATP} \underset{\mathrm{Mg}^{++}}{\stackrel{\text { Gal-Kinase }}{\longrightarrow}} \mathrm{Gal}-1-\mathrm{P}+\mathrm{ADP}$.

Die nichtphosphorylierte Galaktose wird umgesetzt zu 2. $\mathrm{Gal}+\mathrm{NAD}^{+} \stackrel{\mathrm{Gal}-\mathrm{DH}}{\longrightarrow}$ Galaktonolacton $+\mathrm{NADH}+\mathrm{H}^{+}$.

Beim Literaturstudium fanden sich bei den verschiedenen Autoren Gitzelmann $(2,3)$ Mayes \& Guthrie (5), Ng (4), Robinson (6) Abweichungen in der Technik der Isotopenmethode. Deswegen wurden zunächst die angegebenen Versucbsbedingungen überprüft.

1. Det Einfluß der Substratkonzentration auf die Galaktokinaseaktivität wurde untersucht. Aufgrund der äußerst geringen Aktivität der Galaktokinase war anzunehmen, daß das von NG angegebene Substratangebot ausreichend ist.

2. Die unterschiedlich angegebenen $p H$-Optima für die Galaktokinase veranlaßten die Úberprüfung der Empfindlichkeit der Galaktose-Kinase gegen $\mathrm{pH}-\AA$ nderung. Nach diesen Ergebpissen wurde das pH-Optimum neu festgelegt. 


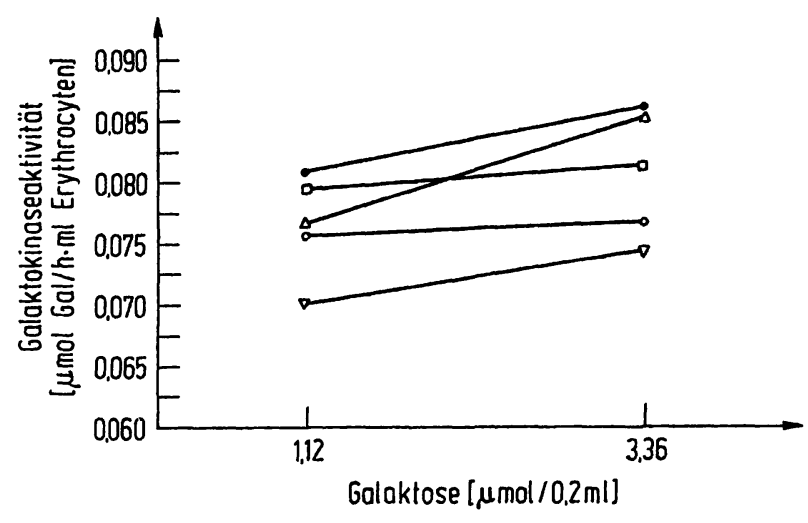

Abb. 1

Steigerung der Galaktokinaseaktivität bei dreifachem Substratangebot. Verwendet wurden Hämolysate von fünf verschiedenen Probanden in den Konzentrationen 1,12 und $3,36 \mu \mathrm{mol} / 0,2 \mathrm{ml}$

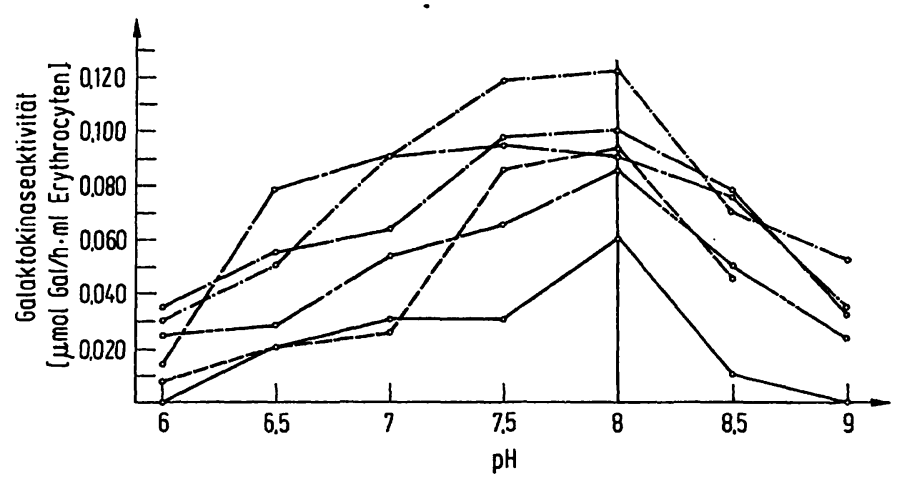

Abb. 2

Galaktokinaseaktivität bei verschiedenen $\mathrm{pH}-$ Werten des verwendeten Tris-Puffers. Die Hämolysate stammen von 6 verschiedenen Probanden. Die durch Kurvenzug miteinander verbundenen Werte wurden jeweils aus dem Hämolysat eines Probanden ermittelt

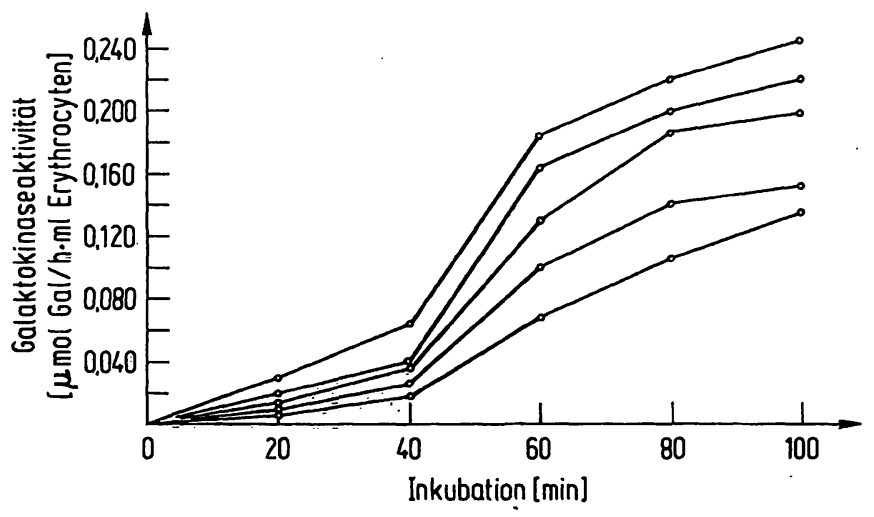

Abb. 3

Die Galaktokinaseaktivität in Abhängigkeit von der Inkubationszeit. Verwendet wurden Hämolysate von 5 verschiedenen Personen

3. Die Angaben der Autoren über die optimale Inkubationszeit erstrecken sich von $30-90 \mathrm{~min}$.

Der Einflu $\beta$ der Inkubationszeit wurde unter vergleichbaren Ansatzbedingungen überprüft. Für den Mittelwert von $60 \mathrm{~min}$ zwischen den Extremen $30 \mathrm{~min}$ (NG) und 90 min (GiTzelmanN) wurde entschieden, da der Reaktionsumsatz offensichtlich eine lange Anlaufzeit benötigt und die 30 Minuten-Marke noch in diesem Bereich liegt. Auf die 90 Minuten-Marke wurde trotz des höheren Umsatzes verzichtet, da die Versuchszeit für die enzymatische Methode kurz gehalten werden sollte.

In allen Ansätzen wurde bei $37,5^{\circ} \mathrm{C}$ inkubiert und mit $\mathrm{Mg}^{++}$ Ionen aktiviert.

Unter Verwendung der Ergebnisse der Voruntersuchungen wurden sowohl für das Isotopenverfahren als auch für die enzymatische Methode folgende Inkubationsbedingungen gewählt:

\section{Reagenzien}

1. Galaktoselösung $5,6 \mathrm{mmol} / 1$.

2. ATP-Lösung $40 \mathrm{mmol} / \mathrm{l}$.

3. $\mathrm{MgSO}_{4}$-Lösung $0,8 \mathrm{mmol} / \mathrm{l}$.

4. Tris-Puffer $0,7 \mathrm{mmol} / \mathrm{l}$ auf $\mathrm{pH} 8,0$ eingestcllt.

5. Galaktose-UV-Test (Fa. Boehringer-Mannheim).

Lagerung und Haltbarkeit

Bei Lagerung im Kühlschrank bei $4^{\circ} \mathrm{C}$ und gutem Flaschenverschluß ist die Lösung 1 bis zu drei Monaten und die Lösung 4 bis zu sechs Wochen haltbar.

Die Lösungen 2 und 3 werden tiefgefroren $\left(-16^{\circ} \mathrm{C}\right)$ gelagert und sind vier bis sechs Monate haltbar.

Der Puffer wird vor jedem Versuch auf Raumtemperatur erwärmt und der $\mathrm{pH}$-Wert überprüft.

Aus arbeitstechnischen Gründen kann es nötig sein, daß das eingesandte Blut gelagert werden muß.

NG (4) gibt einen Abfall der Galaktokinaseaktivität bei Lagerıng des Hämolysates in gefrorenem Zustand an.

Nach diesem Autor gehen über $20 \%$ der Aktivität an einem Tag verloren, $90 \%$ nach sechs Tagen Lagerung.

Eigene Versuche ergaben nach viertägiger Lagerung des Vollblutes bei Raumtemperatır einen 20proz. Aktivitätsverlust.

\section{Vorbereitung des 50proz. Hämolysates}

Das aus der Cubitalvene entnommene Blut wird mit 30-50 E Heparin/ml versetzt. Das heparinisierte Vollblut wird $25 \mathrm{~min}$ bei $2000 \mathrm{~g}$ in der Kälte zentrifugiert.

Das überstehende Plasma wird dekantiert; die obcre Leukocyten-, Erythrocyten- und Thrombocytenschicht (= buffy coat) wird abgehoben. Die abgesetzten Erythrocyten werden durch Suspendieren in kalter, isotonischer $\mathrm{NaCl}$-Lösung und Zentrifugieren in der Kälte zweimal gewaschen. Der Ubberstand wird nach jedem Waschvorgang abpipettiert und verworfen. Die abgesetzten Erythrocyten werden zur Herstellung eines 50proz. Hämolysates mit kaltem, destilliertem Wasser gemischt und für zweimal 4 bis $5 \mathrm{~min}$ in einem Methanol-Bad bei etwa $-28^{\circ} \mathrm{C}$ gefroren und wieder aufgetaut.

\section{Bestimmungsansatz}

$\mathrm{Zu}$ jeder Untersuchung werden 2 Reaktions- und 2 Leerwcrte angesetzt. Die beschrifteten Röhrchen werden in ein Eisbad gestellt und erst dann wird mit dem Einpipettieren der Lösungen begonnen.

Folgende Reagenzien werden für Reaktions- und Leerwert in die Röhrchen pipettiert:

\begin{tabular}{lcc}
\hline \multicolumn{1}{c}{ Reagenz } & Reaktionswert & Leerwert \\
\hline Galaktose-Lösung & $0,2 \mathrm{ml}$ & $0,2 \mathrm{ml}$ \\
ATP-Lösung & $0,2 \mathrm{ml}$ & - \\
MgSO,-Lösung & $0,2 \mathrm{ml}$ & $0,2 \mathrm{ml}$ \\
Tris-Puffer-Lösung & $0,5 \mathrm{ml}$ & $0,5 \mathrm{ml}$ \\
bidest. Wasser & - & $0,2 \mathrm{ml}$ \\
Hämolysat & $1,0 \mathrm{ml}$ & $1,0 \mathrm{ml}$ \\
\hline
\end{tabular}

Nach dem Einpipettieren werden die Röhrchen mit Parafilm verschlossen und sorgfältig durchgemischt. Sofort anschließend wird in einem Wasserbad bei $37,5^{\circ} \mathrm{C}$ für $60 \mathrm{~min}$ inkubiert. Die Röhrchen werden danach für 5 min zurück ins Eisbad gebracht, um dic Reaktion abzustoppen. Zur Hitzekoagulation kommen sie für 2-4 $\mathrm{min}$ in ein siedendes Wasserbad, währenddessen intermittietend geschüttelt wird.

Anschließend werden dic Gläser zum Abkühlen für einige Minuten in das Eisbad gestellt und darauf $15-20 \mathrm{~min}$ bei $3000 \mathrm{~g}$ zentrifugiert.

Bei der Bestimmung nach No et al. folgen jetzt Chromatographie und Scintillationszählung.

Bei der von uns crarbeiteten Methode wird folgender Weg eingeschlagen: 


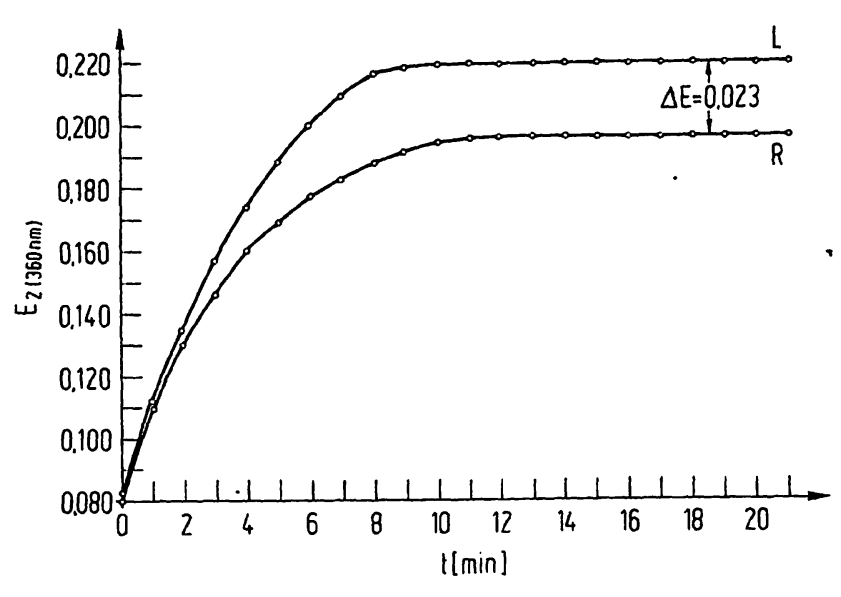

Abb. 4

Extinktionsänderung von Reaktions- und Leerwert nach NAD-Zugabe

Bestimmung der nichtphosphorylierten Galaktose mit der "Galaktose-Biochemica-Test-Kombination" der Fa. Boehringer-Mannheim.

\section{Reagenzien}

1. Puffer (Tris-Puffer, $0,1 \mathrm{~mol} / \mathrm{l} \mathrm{pH} 8,6$ ):

Inhalt der Flasche 1 mit bidest. Wasser auf $100 \mathrm{ml}$ verdünnen. Bei Raumtemperatùr ein Jahr haltbar.

2. $\operatorname{NAD}(0,013 \mathrm{~mol} / \mathrm{l})$ :

Inhalt der Flasche 2 mit $3,3 \mathrm{ml}$ dest. Wasser lösen; bei etwa $4^{\circ} \mathrm{C}$ 4 Wochen haltbar.

3. Galaktose-Dehydrogenase $(5 \mathrm{mg} / \mathrm{ml})$ :

Inhalt der Flasche 3 unverdünnt verwenden. Bei etwa $4^{\circ} \mathrm{C}$ ein Jaht haltbar.

Die Angaben zu den Reagenzien wurden der Testanleitung der $\mathrm{Fa}$. Boehringer entnommen. Ungefähr $20 \mathrm{~min}$ nach Zugabe von Galaktosedehydrogenase ist die enzymatische Oxidation der nichtphosphorylierten Galaktose zum Stillstand gekommen.

Die Differenz zwischen endgültigem Leer- und Reaktionswert läßt sich aus der Abbildung 4 bestimmen, oder aus der Rechnung, die im Folgenden beschrieben wird:

\section{Berechnung}

Grundlage für die Berechnung der Enzymaktivität ist der mikromolare Extinktionskoeffizient für NAD.

Es gilt:

$\Delta \mathrm{E} \cdot \mathrm{K} \cdot 2=\mu \mathrm{mol}$ phosphorylierte Galaktose $/ \mathrm{h} \cdot \mathrm{ml}$ Erythrocyten.

Die Extinktionsdifferenz $\Delta \mathrm{E}$ ergibt sich aus:

$\Delta \mathrm{E}=\left(\mathrm{E}_{2}-\mathrm{E}_{1}-\mathrm{E}_{0}\right)_{\text {Leerwert }}-\left(\mathrm{E}_{2}-\mathrm{E}_{1}-\mathrm{E}_{0}\right)_{\text {Reaktionswert }}$

$\mathrm{E}_{0}=$ Eigenextinktion der Galaktosedehydrogenase

$E_{1}=$ Anfangsextinktion von Reaktions- und Leeransatz

$\mathrm{E}_{2}=$ Extinktion nach Zugabe der Galaktosedehydrogenase.

Die Konstante $\mathrm{K}$ setzt sich zusammen aus:

$\mathrm{K}=\frac{\mathrm{V} \cdot \mathrm{F}}{\varepsilon \cdot \mathrm{d} \cdot \mathrm{V} \cdot \mathrm{T}}=10,5$

$\mathrm{V}=$ Testvolumen in Küvette $(3,32 \mathrm{ml})$

$\mathrm{F}=$ Verdünnungsfaktor für Hämolysat $(2,1)$

$\varepsilon=$ mikromolarer Extinktionskoeffizient von $\operatorname{NAD}\left(3,3 \mathrm{~cm}^{2} /\right.$ $\mu \mathrm{mol}$ bei $366 \mathrm{~nm}$ )

d $=$ Schichtdicke i $1 \mathrm{~cm}(1 \mathrm{~cm})$

$\nabla=0,2 \mathrm{ml}$ Filtratvolumen

$\mathrm{T}=$ Inkubationszeit $(1 \mathrm{~h})$.

Der Faktor 2 der Berechnungsformel ergibt sich aus der 1:2 Verdünnung der Erythrocyten zur Hämolyse.

\section{Ergebnisse}

\section{Normalwerte}

Es wurden Blutproben von einem Normalkollektiv von 33 Erwachsenen und 6 Säuglingen untersucht.

Die Galaktokinase-Aktivität bei Adulten schwankt zwischen 0,431 und $0,615 \mu \mathrm{mol}$ phosphorylierter Galaktose $/ \mathrm{h} \cdot \mathrm{ml}$ Erythrocyten $\left(\overline{\mathrm{x}}=0,543 ; \mathrm{s}_{\mathrm{x}}=0,048 \mu \mathrm{mol}\right.$ phosphorylierte Galaktose/h $\cdot \mathrm{ml}$ Erythrocyten).

Die Galaktokinase-Aktivität bei Säuglingen schwankt $z$ wischen 0,750 und $0,835 \mu$ mòl phosphorylierter Galaktose $/ \mathrm{h} \cdot \mathrm{ml}$ Erythrocyten ( $\overrightarrow{\mathrm{x}}=0,797 ; \mathrm{s}_{\mathbf{x}}=0,026 \mu \mathrm{mol}$ phosphorylierte Galaktose/h $\cdot \mathrm{ml}$ Erythrocyten).

\section{Reproduzierbarkeit der Ergebnisse}

Ausgehend von der Blutprobe eines gesunden Erwachsenen wurden an einem Tag 1.0 Einzelbestimmungen der Galaktokinase-Aktivität durchgeführt. Bei $\overline{\mathrm{x}}=0,562$; $\mathrm{s}_{\mathrm{x}}=0,025 \mu \mathrm{mol}$ phosphorylierter Galaktose $/ \mathrm{h} \cdot \mathrm{ml}$ Erythrocyten fanden sich Extremwerte von 0,545 und 0,606 $\mu$ mol phosphorylierter Galaktose/h $\cdot \mathrm{ml}$ Erythrocyten. Der Variationskoeffizient betrug 4,5\%.

Vom Blut zweier weiterer Probanden wurden zusätzlich 10 Einzelbestimmungen an einem Tag durchgeführt.

Der Mittelwert der Variationskoeffizienten aller drei Untersuchungen lag bei 5,06\%.

Wird die Streuung der Einzelergebnisse vom Mittelwert betrachtet, dann ist der methodische Fehler nicht so groß, als daß nicht eine deutliche Trennung von möglichen Heterozygotenwerten zu ziehen wäre.

Vergleichende Aktivitätsbestimmungen bei einem Normalkollektiv von Adulten und Säuglingen

Insgesamt wurden 39 Hämolysạte von gesunden Probanden untersishiedlichen Alters untersucht.

Von diesen Hämolysaten stammen 33 von gesunden Erwachsenen im Alter von 20-35 Jahren und 6 von Säuglingen im Alter von 6-10 Monaten.

Anamnestisch und klinisch bestand bei keinem der Probanden ein Hinweis auf Uridylyltransferasemangel nọch auf Galaktokinasemangel.

Die Werte der Galaktokinaseaktivität der Probanden sind in Abbildung 5 dargestellt.

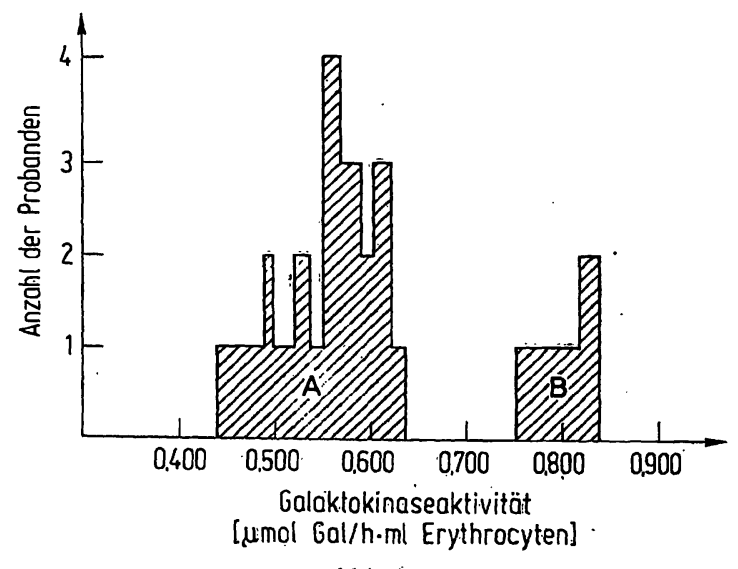

Abb. 5

Galaktokinaseaktivität von (A) 33 gesunden Erwachsenen im Alter von $20-35$ Jahren und (B) 6 Säuglingen im Alter von 6-10 Monaten 
Obwohl sich eine ausgeprägte Schwankung der Werte innerhalb der Altersklassen von 20-35 Jahren ergibt, stellt sich die höhere Aktivität der Säuglinge deutlich dar, die im Mittel um 47\% über der von Erwachsenen liegt.

Ergebnis der Aktivitätsbestimmung mit Hilfe der Isotopenmethode nach NG - DonNell BERGREN

Aus den genannten Gründen wurden die Bedingungen für den Inkubationsansatz nach NG et al. modifiziert und mit denen für das enzymatische Verfahren gleichgesetzt.

Nun erst wurden vergleichende Galaktokinaseaktivitätsbestimmungen eines Hämolysates mit beiden Methoden ausgeführt. Aus arbeitstechnischen Gründen war es nur möglich, die Kinaseaktivität von 6 verschiedenen Probanden im Alter von 20-35 Jahren mit beiden Verfahren zu messen (Tab. 1).

Bei diesen Untersuchungen zeigte sich, wie abhängig man bei dem Verfahren nach NG et al. von'der Belastung und Funktionstüchtigkeit des Scintillationszählers ist.

\section{Diskussion}

Die von uns erstellte Methode zur Bestimmung der Galaktokinaseaktivität ergab höhere Werte sowohl nach dem ursprünglichen (vgl. Tab. 2) als auch nach dem modifizierten NG-Verfahren (siehe Tab. 1). Grundsätzlich sind die höheren Umsatzwerte auf die Modifikation der Inkubationszeit des $\mathrm{pH}$-Wertes des Puffers zurückzuführen.

Vergleicht man die Zahlen von Tabelle 1, so zeigt sich eine deutliche Differenz zwischen den Mittelwerten der NG-Methode (modifiziert) und den Mittelwerten des eigenen Verfahrens. Das für beide Methoden benötigte Filtrat wurde aus dem gleichen Inkubationsansatz entnommen.

Tab. 1

Ergebnis der Vergleichsbestimmungen

\begin{tabular}{cc}
$\begin{array}{c}\text { NG-Methode (modifiziert) } \\
{[\mu \mathrm{mol} \text { phosphorylierte Galaktose/h } \cdot \text { mI Erythrocyten }]}\end{array}$ & $\begin{array}{c}\text { Enzymatische Methode } \\
\text { O,400 }\end{array}$ \\
0,394 & 0,537 \\
0,394 & 0,494 \\
0,282 & 0,516 \\
0,448 & 0,431 \\
0,324 & 0,545 \\
\hline$\overline{\mathbf{x}}=0,373$ & 0,466 \\
\hline
\end{tabular}

Tab. 2

Galaktokinaseaktivitätswerte bei den verschiedenen Altoren

Aktivität

[/mol phosphorylierte Galaktose $/ \mathrm{h} \cdot \mathrm{ml}$ Erythrocyten]

Autoren

\begin{tabular}{ll}
\hline GITZELMANN (3) & $0,120-0,360$ \\
MAYES Und GUTHRIE (5) & $0,234-0,591$ \\
NG et al. (4) & $0,170-0,420$ \\
Eigene Methode & $0,431-0,615$ \\
\hline
\end{tabular}

Möglicherweise erklärt sich die höhere Umsatzmessung der enzymatischen Methode gegenüber der radioaktiven Messung dadurch, da $\beta$ der Scintillationszähler die Lichtblitze des Filtrats in der angewandten Meßzeit von 10 min nicht vollständig erfaßt.

Die Auswertung der eigenen Versuche mit der enzymatischen Methode bestätigt die Aussagen von NG et al. über den bedeutend höheren Umsatz bei Säuglingen, sowie über eine Abnahme der Kinaseaktivität mit zunehmendem Alter und die individuellen Schwankungen innerhalb einer Altersgruppe.

KRAUSS und MöNCH (7) geben einen enzymatischen zusammengesetzten Test an, durch den die katalytische Aktivität der Kinase in Erythrocyten bestimmt werden kann. Die Bildung von Galaktose-1-phosphat aus Galaktose und ATP wird durch Zusatz von Uridylyltransferase, Phosphoglucomutase und Glucose-6-phosphat-Dehydrogenase im direkten optischen Test gemessen. Die Autoren beschicken die Küvetten mit nicht definierten Mengen von Hämolysat. Bei ihnen findet sich keine Angabe über den Störeffekt des Hämoglobins und über die sehr hohen Enzymaktivitäten.

Die kürzlich veröffentlichte Arbeit von KaLoud und Sirzmann (8) über einen enzymatischen Test zur Bestimmung der Kinaseaktivität enthält sehr hohe Aktivitätswerte $(0,3-3 \mu \mathrm{mol} / \mathrm{h} \cdot \mathrm{g} \mathrm{Hb})$ mit großer Schwankungsbreite.

Das Prinzip dieser Autoren beruht auf einer gekoppelten enzymatischen Reaktion, in der die Bildung von Galaktose-1-phosphat aus Galaktose und ATP durch Zusatz von Phosphoenolpyruvat, Pyruvatkinase, Lactatdehydrogenase und $\mathrm{NADH}$ im direkten optischen Test verfolgt werden kann.

Im Gegensatz zu den Methoden der anderen Autoren, die ihre optische Aktivitätsmessung erst nach Zugabe mehrerer Enzyme durchführen, wird in unserem Verfahren die nichtphosphorylierte Galaktose unmittelbar mit der Galaktosedehydrogenase bestimmt. Zusätzliche Fehlerquellen werden somit vermieden.

\section{Literatur}

1. Kalckar, H. M. \& ANderson, E. P. (1956), Biochim. Biophys. Acta 20, 262-268. - 2. GirzeLmanN, R. (1965), Lancet II, 670-676. - 3. Grtzelmann, R. (1967), Pediat. Res. 1, 14-23. 4. NG, Won. G. \& Donneli, G. N. (1965), J. Lab. Clin. Med. 66, 115-121. - 5. Mayes, J. S. \& Guthrie, R. (1968), Biochem.
Genet. 2, 219-230. - 6. Rournson, A. (1963), J. Exp. Med. 118 , 359-370. - 7. Kraus, H. \& MönCh, E. (1970), Klin. Wochenschr. 48, 437-439. - 8. KALoud, H. \& SitzManN, F. C. (1971), Beiheft z. Arch. Kinderheilk. 64. 